See discussions, stats, and author profiles for this publication at: https://www.researchgate.net/publication/2109398

\title{
Sturm Oscillation and Comparison Theorems
}

Article · December 2003

DOI: $10.1007 / 3-7643-7359-8 \_2 \cdot$ Source: arXiv

CITATIONS

READS

30

711

1 author:

Barry Simon

6 C California Institute of Technology

537 PUBLICATIONS 48,989 CITATIONS

SEE PROFILE 


\title{
STURM OSCILLATION AND COMPARISON THEOREMS
}

\author{
BARRY SIMON
}

\begin{abstract}
This is a celebratory and pedagogical discussion of Sturm oscillation theory. Included is the discussion of the difference equation case via determinants and a renormalized oscillation theorem of Gesztesy, Teschl, and the author.
\end{abstract}

\section{INTRODUCTION}

Sturm's greatest contribution is undoubtedly the introduction and focus on Sturm-Liouville operators. But his mathematically deepest results are clearly the oscillation and comparison theorems. In [22, 23, he discussed these results for Sturm-Liouville operators. There has been speculation that in his unpublished papers he had the result also for difference equations, since shortly before his work on Sturm-Liouville operators, he was writing about zeros of polynomials, and there is a brief note referring to a never published manuscript that suggests he had a result for difference equations. Indeed, the Sturm oscillation theorems for difference equations written in terms of orthogonal polynomials are clearly related to Descartes' theorem on zeros and sign changes of coefficients.

In any event, the oscillation theorems for difference equations seem to have appeared in print only in 1898 [2], and the usual proof given these days is by linear interpolation and reduction to the ODE result. One of our purposes here is to make propaganda for the approach via determinants and orthogonal polynomials (see Section 22). Our discussion in Section 3 and 4 is more standard ODE theory [3] — put here to have a brief pedagogical discussion in one place. Section 5 makes propaganda for what I regard as some interesting ideas of Gesztesy, Teschl, and me [8]. Section [6] has three applications to illustrate the scope of applicability.

Date: October 15, 2003.

${ }^{1}$ Mathematics 253-37, California Institute of Technology, Pasadena, CA 91125. E-mail: bsimon@caltech.edu. Supported in part by NSF grant DMS-0140592. 
Our purpose here is celebratory and pedagogical, so we make simplifying assumptions, such as only discussing bounded and continuous perturbations. Standard modern techniques allow one to discuss much more general perturbations, but this is not the place to make that precise. And we look at Schrödinger operators, rather than the more general Sturm-Liouville operators.

We study the ODE

$$
H u=-\frac{d^{2} u}{d x^{2}}+V u=E u
$$

typically on $[0, a]$ with $u(0)=u(a)=0$ boundary conditions or on $[0, \infty)$ with $u(0)=0$ boundary conditions. The discrete analog is

$$
(h u)_{n}=a_{n} u_{n+1}+b_{n} u_{n}+a_{n-1} u_{n-1}=E u
$$

for $n=1,2, \ldots$ with $u_{0} \equiv 0$.

It is a pleasure to thank W. Amrein for the invitation to give this talk and for organizing an interesting conference, Y. Last and G. Kilai for the hospitality of Hebrew University where this paper was written, and F. Gesztesy for useful comments.

\section{Determinants, Orthogonal Polynomials, and Sturm TheORY FOR DiffERENCE EqUATIONS}

Given a sequence of parameters $a_{1}, a_{2}, \ldots$ and $b_{1}, b_{2}$ for the difference equation (1.2), we look at the fundamental solution, $u_{n}(E)$, defined recursively by $u_{1}(E)=1$ and

$$
a_{n} u_{n+1}(E)+\left(b_{n}-E\right) u_{n}(E)+a_{n-1} u_{n-1}(E)=0
$$

with $u_{0} \equiv 0$, so

$$
u_{n+1}(E)=a_{n}^{-1}\left(E-b_{n}\right) u_{n}(E)-a_{n}^{-1} a_{n-1} u_{n-1}(E)
$$

Clearly, (2.2) implies, by induction, that $u_{n+1}$ is a polynomial of degree $n$ with leading term $\left(a_{n} \ldots a_{1}\right)^{-1} E^{n}$. Thus, we define for $n=0,1,2, \ldots$

$$
p_{n}(E)=u_{n+1}(E) \quad P_{n}(E)=\left(a_{1} \ldots a_{n}\right) p_{n}(E)
$$

Then (2.1) becomes

$$
a_{n+1} p_{n+1}(E)+\left(b_{n+1}-E\right) p_{n}(E)+a_{n} p_{n-1}(E)=0
$$

for $n=0,1,2, \ldots$ One also sees that

$$
E P_{n}(E)=P_{n+1}(E)+b_{n+1}(E) P_{n}(E)+a_{n}^{2} P_{n-1}(E)
$$


We will eventually see $p_{n}$ are orthonormal polynomials for a suitable measure on $\mathbb{R}$ and the $P_{n}$ are what are known as monic orthogonal polynomials.

Let $J_{n}$ be the finite $n \times n$ matrix

$$
J_{n}=\left(\begin{array}{cccccc}
b_{1} & a_{1} & 0 & & & \\
a_{1} & b_{2} & a_{2} & & & \\
0 & a_{2} & b_{3} & \ddots & & \\
& & \ddots & \ddots & \ddots & \\
& & & \ddots & b_{n-1} & a_{n-1} \\
& & & & a_{n-1} & b_{n}
\end{array}\right)
$$

Proposition 2.1. The eigenvalues of $J_{n}$ are precisely the zeros of $p_{n}(E)$. We have

$$
P_{n}(E)=\operatorname{det}\left(E-J_{n}\right)
$$

Proof. Let $\varphi(E)$ be the vector $\varphi_{j}(E)=p_{j-1}(E), j=1, \ldots, n$. Then (2.1) implies

$$
\left(J_{n}-E\right) \varphi(E)=-a_{n} p_{n}(E) \delta_{n}
$$

where $\delta_{n}$ is the vector $(0,0, \ldots, 0,1)$. Thus every zero of $p_{n}$ is an eigenvalue of $J_{n}$. Conversely, if $\tilde{\varphi}$ is an eigenvector of $J_{n}$, then both $\tilde{\varphi}_{j}$ and $\varphi_{j}$ solve (2.2), so $\tilde{\varphi}_{j}=\tilde{\varphi}_{1} \varphi_{j}(E)$. This implies that $E$ is an eigenvalue only if $p_{n}(E)$ is zero and that eigenvalues are simple.

Since $J_{n}$ is real symmetric and eigenvalues are simple, $p_{n}(E)$ has $n$ distinct eigenvalues $E_{j}^{(n)}, j=1, \ldots, n$ with $E_{j-1}^{(n)}<E_{j}^{(n)}$. Thus, since $p_{n}$ and $P_{n}$ have the same zeros,

$$
P_{n}(E)=\prod_{j=1}^{n}\left(E-E_{j}^{(n)}\right)=\operatorname{det}\left(E-J_{n}\right)
$$

Proposition 2.2. (i) The eigenvalues of $J_{n}$ and $J_{n+1}$ strictly interlace, that is,

$$
E_{1}^{(n+1)}<E_{1}^{(n)}<E_{2}^{(n+1)}<\cdots<E_{n}^{(n)}<E_{n+1}^{(n+1)}
$$

(ii) The zeros of $p_{n}(E)$ are simple, all real, and strictly interlace those of $p_{n+1}(E)$.

Proof. (i) $J_{n}$ is obtained from $J_{n+1}$ by restricting the quadratic form $u \rightarrow\left\langle u, J_{n+1} u\right\rangle$ to $\mathbb{C}^{n}$, a subspace. It follows that $E_{1}^{(n+1)}=$ 
$\min _{u,\|u\|=1}\left\langle u, J_{n+1} u\right\rangle \leq \min _{u \in \mathbb{C}^{n},\|u\|=1}\left\langle u, J_{n+1} u\right\rangle=E_{1}^{(n)}$. More generally, using that min-max principle

$$
E_{j}^{(n+1)}=\max _{\varphi_{1}, \ldots, \varphi_{j-1}} \min _{\substack{\|u\|=1 \\ u \perp \varphi_{1}, \ldots, \varphi_{j-1}}}\left\langle u, J_{n+1} u\right\rangle
$$

one sees that

$$
E_{j}^{(n)} \geq E_{j}^{(n+1)}
$$

By replacing min's with max's,

$$
E_{j}^{(n)} \leq E_{j+1}^{(n+1)}
$$

All that remains is to show that equality is impossible. If $E_{0} \equiv$ $E_{j}^{(n)}=E_{j}^{(n+1)}$ or $E_{0} \equiv E_{j}^{(n)}=E_{j}^{(n+1)}$, then $p_{n+1}\left(E_{0}\right)=p_{n}\left(E_{0}\right)=0$. By (2.4), this implies $p_{n-1}\left(E_{0}\right)=0$ so, by induction, $p_{0}(E)=0$. But $p_{0} \equiv 1$. Thus equality is impossible.

(ii) Given (2.6), a restatement of what we have proven about the eigenvalues of $J_{n}$.

Here is our first version of Sturm oscillation theorems:

Theorem 2.3. Suppose $E_{0}$ is not an eigenvalue of $J_{k}$ for $k=$ $1,2, \ldots, n$. Then

$$
\begin{aligned}
& \#\left(j \mid E_{j}^{(n)}>E_{0}\right)=\#\left\{\ell=1, \ldots, n \mid \operatorname{sgn}\left(P_{\ell-1}\left(E_{0}\right)\right) \neq \operatorname{sgn}\left(P_{\ell}\left(E_{0}\right)\right)\right\} \\
& \#\left(j \mid E_{j}^{(n)}<E_{0}\right)=\#\left\{\ell=1, \ldots, n \mid \operatorname{sgn}\left(P_{\ell-1}\left(E_{0}\right)=\operatorname{sgn}\left(P_{\ell}\left(E_{0}\right)\right)\right\}\right.
\end{aligned}
$$

Proof. (2.9) clearly implies (2.10) since the sum of both sides of the equalities is $n$. Thus we need only prove (2.9).

Suppose that $E_{1}^{(\ell)}<\cdots<E_{k}^{(\ell)}<E_{0}<E_{k+1}^{(\ell)}<E_{n}^{(\ell)}$. By eigenvalue interlacing, $J_{\ell+1}$ has $k$ eigenvalues in $\left(-\infty, E_{k}^{(\ell)}\right)$ and $n-k$ eigenvalues in $\left(E_{k+1}^{(\ell)}, \infty\right)$. The question is whether the eigenvalue in $\left(E_{k}^{(\ell)}, E_{k+1}^{(\ell)}\right)$ lies above $E_{0}$ or below. Since $\operatorname{sgn} \operatorname{det}\left(E-J^{(\ell+1)}\right)=(-1)^{\#\left(j \mid E_{j}^{(\ell)}>E_{0}\right)}$, and similarly for $J_{\ell+1}$, and there is at most one extra eigenvalue above $E_{0}$, we see

$$
\begin{aligned}
& \operatorname{sgn} P_{\ell}\left(E_{0}\right)=\operatorname{sgn} P_{\ell+1}\left(E_{0}\right) \Leftrightarrow \#\left(j \mid E_{j}^{(\ell)}>E_{0}\right)=\#\left(j \mid E_{j}^{(\ell+1)}>E_{0}\right) \\
& \operatorname{sgn} P_{\ell}\left(E_{0}\right)=\operatorname{sgn} P_{\ell+1}\left(E_{0}\right) \Leftrightarrow \# j\left(\mid E_{j}^{(\ell)}>E_{0}\right)+1=\#\left(j \mid E_{j}^{(\ell+1)}>E_{0}\right)
\end{aligned}
$$

(2.9) follows from this by induction. 
We want to extend this in two ways. First, we can allow $P_{k}\left(z_{0}\right)=0$ for some $k<n$. In that case, by eigenvalue interlacing, it is easy to see $J_{k+1}$ has one more eigenvalue than $J_{k-1}$ in $\left(E_{0}, \infty\right)$ and also in $\left(-\infty, E_{0}\right)$, so $\operatorname{sgn}\left(P_{k-1}\left(z_{0}\right)\right)=-\operatorname{sgn}\left(P_{k+1}\left(z_{0}\right)\right)$ (also evident from (2.5) and $\left.P_{k}\left(z_{0}\right)=0\right)$. Thus we need to be sure to count the change of sign from $<0,0$ to $>0, a$ as only a simple change of sign. We therefore have

Proposition 2.4. (2.9) and (2.10) remain true so long as $P_{n}\left(E_{0}\right) \neq 0$ so long as we define $\operatorname{sgn}(0)=1$. If $P_{n}\left(E_{0}\right)=0$, they remain true so long as $\ell=n$ is dropped from the right side.

One can summarize this result as follows: For $x \in[0, n]$, define $y(x)$ by linear interpolation, that is,

$$
x=[x]+(x) \Rightarrow y(x)=P_{[x]}+(x)\left(P_{[x]+1}-P_{[x]}\right)
$$

Then the number of eigenvalues of $J_{n}$ above $E$ is the number of zeros of $y(x, E)$ in $[0, n)$. If we do the same for $\tilde{y}$ with $P_{[x]}$ replaced by $(-1)^{[x]} P_{[x]}$, then the number of eigenvalues below $E$ is the number of zeros of $\tilde{y}$ in $[0, n)$. Some proofs (see [5]) of oscillation theory for difference equations use $y$ and mimic the continuum proof of the next section.

The second extension involves infinite Jacobi matrices. In discussing eigenvalues of an infinite $J$, domain issues arise if $J$ is not bounded (if the moment problem is not determinate, these are complicated issues; see Simon [21]). Thus, let us suppose

$$
\sup _{n}\left(\left|a_{n}\right|+\left|b_{n}\right|\right)<\infty
$$

If $J$ is bounded, the quadratic form of $J_{n}$ is a restriction of $J$ to $\mathbb{C}^{n}$. As in the argument about eigenvalues interlacing, one shows that if $J$ has only $N_{0}<\infty$ eigenvalues in $\left(E_{0}, \infty\right)$, then $J_{n}$ has at most $N_{0}$ eigenvalues there. Put differently, if $E_{1}^{(\infty)}>E_{2}^{(\infty)}>\cdots$ are the eigenvalues of $J, E_{j}^{(\infty)} \geq E_{j}^{(n)}$. Thus, if $N_{n}(E)=\#$ of eigenvalues of $J_{n}$ in $(E, \infty)$ and $N_{\infty}$ the dimension of $\operatorname{Ran} P_{(E, \infty)}(J)$, the spectral projection

$$
N_{n}(E) \leq N_{n+1}(E) \leq \cdots \leq N_{\infty}(E)
$$

On the other hand, suppose we can find an orthonormal set $\left\{\varphi_{j}\right\}_{j=1}^{N}$ with $M_{j k}^{(\infty)}=\left\langle\varphi_{j}, J \varphi_{k}\right\rangle=e_{j} \delta_{j k}$ and $\min \left(e_{j}\right)=e_{0}>E_{0}$. If $M_{j k}^{(n)}=$ $\left\langle\varphi_{j}, J_{n} \varphi_{k}\right\rangle, M^{(n)} \rightarrow M^{(\infty)}$, so for $n$ large, $M^{(n)} \geq \min \left(e_{j}\right)+\frac{1}{2}\left(e_{0}-E_{0}\right)>$ $E_{0}$. Thus $N_{n}\left(E_{0}\right) \geq N$ for $n$ large. It follows that $\lim N_{n} \geq N_{\infty}$, that is, we have shown that $N_{\infty}\left(E_{0}\right)=\lim _{n \rightarrow \infty} N_{n}\left(E_{0}\right)$. Thus, 
Theorem 2.5. Let $J$ be an infinite Jacobi matrix with (2.11). Then (with $\operatorname{sgn}(0)=1$ ) we have

$$
\begin{aligned}
N_{\infty}\left(E_{0}\right) & =\#\left\{\ell=1,2, \ldots \mid \operatorname{sgn}\left(P_{\ell-1}\left(E_{0}\right)\right) \neq \operatorname{sgn}\left(P_{\ell}\left(E_{0}\right)\right)\right\} \\
\operatorname{dim} P_{\left(-\infty, E_{0}\right)}(J) & =\#\left\{\ell=1,2, \ldots \mid \operatorname{sgn}\left(P_{\ell-1}\left(E_{0}\right)\right)=\operatorname{sgn}\left(P_{\ell}\left(E_{0}\right)\right)\right\}
\end{aligned}
$$

Corollary 2.6. $a_{-} \leq J \leq a_{+}$if and only if for all $\ell$,

$$
P_{\ell}\left(a_{+}\right)>0 \quad \text { and } \quad(-1)^{\ell} P_{\ell}\left(a_{-}\right)>0
$$

While on the subject of determinants and Jacobi matrices, I would be remiss if I did not make two further remarks.

Given (2.6), (2.5) is an interesting relation among determinants, and you should not be surprised it has a determinantal proof. The matrix $J_{n+1}$ has $b_{n+1}$ and $a_{n}$ in its bottom row. The minor of $E-b_{n+1}$ in $E-J_{n+1}$ is clearly $\operatorname{det}\left(E-J_{n}\right)$. A little thought shows the minor of $-a_{n}$ is $-a_{n} \operatorname{det}\left(E-J_{n-1}\right)$. Thus

$$
\operatorname{det}\left(E-J_{n+1}\right)=\left(E-b_{n+1}\right) \operatorname{det}\left(E-J_{n}\right)-a_{n}^{2} \operatorname{det}\left(E-J_{n-1}\right)
$$

which is just (2.5).

Secondly, one can look at determinants where we peel off the top and left rather than the right and bottom. Let $J^{(1)}, J^{(2)}$ be the Jacobi matrices obtained from $J$ by removing the first row and column, the first two, ... Making the $J$-dependence of $P_{n}(\cdot)$ explicit, Cramer's rule implies

$$
\left(z-J_{n}\right)_{11}^{-1}=\frac{P_{n-1}\left(z, J^{(1)}\right)}{P_{n}(z, J)}
$$

In the OP literature, $a_{1}^{-1} p_{n}\left(z, J^{(1)}\right)$ are called the second kind polynomials.

The analog of (2.16) is

$$
P_{n}(z, J)=\left(z-b_{1}\right) P_{n-1}\left(z, J^{(1)}\right)-a_{1}^{2} P_{n-2}\left(z, J^{(2)}\right)
$$

which, by (2.17), becomes

$$
\left[(z-J)_{11}^{-1}\right]^{-1}=\frac{1}{\left(z-b_{1}\right)-a_{1}^{2}\left(z-J_{n-1}^{(1)}\right)_{11}^{-1}}
$$

In particular, since $d \gamma$ is the spectral measure of $\delta_{1}, J$, we have

$$
(z-J)_{11}^{-1}=\int \frac{d \gamma(x)}{z-x} \equiv-m(z, J)
$$


and (2.18) becomes in the limit with $\left(z-J^{(1)}\right)_{11}^{-1} \rightarrow-m\left(z, J^{(1)}\right)$

$$
m(z ; J)=\frac{1}{b_{1}-z-a_{1}^{2} m\left(z ; J^{(1)}\right)}
$$

(2.18) leads to a finite continued fraction expansion of $\left(z-J_{n}\right)_{11}^{-1}$ due to Jacobi, and (2.20) to the Stieltjes continued fraction. Sturm's celebrated paper on zeros of polynomials is essentially also a continued fraction expansion. It would be interesting to know how much Sturm and Jacobi knew of each other's work. Jacobi visited Paris in 1829 (see James [10]), but I have no idea if he and Sturm met at that time.

\section{Sturm Theory of the Real Line}

We will suppose $V$ is a bounded function $[0, \infty)$. We are interested in solutions of

$$
-u^{\prime \prime}+V u=E u
$$

for $E$ real.

Theorem 3.1 (Sturm Comparison Theorem). For $j=1,2$, let $u_{j}$ be not identically zero and solve $-u_{j}^{\prime \prime}+V u_{j}=E_{j} u_{j}$. Suppose $a<b$, $u_{1}(a)=u_{1}(b)=0$ and $E_{2}>E_{1}$. Then $u_{2}$ has a zero in $(a, b)$. If $E_{2}=E_{1}$ and $u_{2}(a) \neq 0$, then $u_{2}$ has a zero in $(a, b)$.

Proof. Define the Wronskian

$$
W(x)=u_{1}^{\prime}(x) u_{2}(x)-u_{1}(x) u_{2}^{\prime}(x)
$$

Then

$$
W^{\prime}(x)=\left(E_{2}-E_{1}\right) u_{1}(x) u_{2}(x)
$$

Without loss, suppose $a$ and $b$ are successive zeros of $u_{1}$. By changing signs of $u$ if need be, we can suppose $u_{1}>0$ on $(a, b)$ and $u_{2}>0$ on $(a, a+\varepsilon)$ for some $\varepsilon$. Thus $W(a)=u_{1}^{\prime}(a) u_{2}(a) \geq 0$ (and, in case $E_{1}=E_{2}$ and $\left.u_{2}(a) \neq 0, W(a)>0\right)$. If $u_{2}$ is nonvanishing in $(a, b)$, then $u_{2} \geq 0$ there, so $W(b)>0$ (if $E_{2}>E_{1},\left(E_{2}-E_{1}\right) \int_{a}^{b} u_{1} u_{2} d x>0$, and if $E_{2}=E_{1}$ but $\left.u_{2}(a) \neq 0, W(a)>0\right)$. Since $W(b)=u_{1}^{\prime}(b) u_{2}(b)$ with $u_{1}^{\prime}(b)<0$ and $u_{2}(b) \geq 0$, this is impossible. Thus we have the result by contradiction.

Corollary 3.2. Let $u(x, E)$ be the solution of (3.1) with $u(0, E)=0$, $u^{\prime}(0, E)=1$. Let $N(a, E)$ be the number of zeros of $u(x, E)$ in $(0, a)$. Then, if $E_{2}>E_{1}$, we have $N\left(a, E_{2}\right) \geq N\left(a, E_{1}\right)$ for all $a$.

Proof. If $n=N\left(a, E_{1}\right)$ and $0<x_{1}<\cdots<x_{n}<a$ are the zeros of $u\left(x, E_{1}\right)$, then, by the theorem, $u\left(x, E_{2}\right)$ has zeros in $\left(0, x_{1}\right),\left(x_{1}, x_{2}\right), \ldots,\left(x_{n-1}, x_{n}\right)$. 
This gives us the first version of the Sturm oscillation theorem:

Theorem 3.3. Let $E_{0}<E_{1}<\cdots$ be the eigenvalues of $H \equiv-\frac{d^{2}}{d x^{2}}+$ $V(x)$ on $L^{2}(0, a)$ with boundary conditions $u(0)=u(a)=0$. Then $u\left(x, E_{n}\right)$ has exactly $n$ zeros in $(0, a)$.

Proof. If $u_{k} \equiv u\left(\cdot, E_{k}\right)$ has $m$ zeros $x_{1}<x_{2}<\cdots x_{m}$ in $(0, a)$, then for any $E>E_{k}, u(\cdot, E)$ has zeros in $(0, x), \ldots,\left(x_{m-1}, x_{m}\right),\left(x_{m}, a\right)$ and so, $u_{k+1}$ has at least $m+1$ zeros. It follows by induction that $u_{n}$ has at least $n$ zeros, that is, $m \geq n$.

Suppose $u_{n}$ has $m$ zeros $x_{1}<\cdots<x_{m}$ in $(0, a)$. Let $v_{0}, \ldots, v_{m}$ be the function $u_{n}$ restricted successively to $\left(0, x_{1}\right),\left(x_{1}, x_{2}\right), \ldots,\left(x_{m}, a\right)$. The $v$ 's are continuous and piecewise $C^{1}$ with $v_{\ell}(0)=v_{\ell}(a)=0$. Thus they lie in the quadratic form domain of $H$ (see [16, 17] for discussions of quadratic forms) and

$$
\begin{aligned}
\left\langle v_{j}, H v_{k}\right\rangle & =\int_{0}^{a} v_{j}^{\prime} v_{k}^{\prime}+\int_{0}^{a} V v_{j} v_{k} \\
& =\delta_{j k} E \int_{0}^{a} v_{j}^{2} d x
\end{aligned}
$$

since if $j=k$, we can integrate by parts and use $-u^{\prime \prime}+V u=E u$.

It follows that for any $v$ in the span of $v_{j}$ 's, $\langle v, H v\rangle=E\|v\|^{2}$, so by the variational principle, $H$ has at least $m+1$ eigenvalues in $\left(-\infty, E_{n}\right)$, that is, $n+1 \geq m+1$.

Remark. The second half of this argument is due to Courant-Hilbert [4].

If we combine this result with Corollary [3.2, we immediately have:

Theorem 3.4 (Sturm Oscillation Theorem). The number of eigenvalues of $H$ strictly below $E$ is exactly the number of zeros of $u(x, E)$ in $(0, a)$.

As in the discrete case, if $H_{a}$ is $-\frac{d^{2}}{d x^{2}}+V(x)$ on $[0, a]$ with $u(0)=$ $u(a)=0$ boundary conditions and $H_{\infty}$ is the operator on $L^{2}(0, \infty)$ with $u(0)=0$ boundary conditions, and if $N_{a}(E)=\operatorname{dim} P_{(-\infty, E)}\left(H_{a}\right)$, then $N_{a}(E) \rightarrow N_{\infty}(E)$, so

Theorem 3.5. The number of eigenvalues of $H_{\infty}$ strictly below $E$, more generally $\operatorname{dim} P_{(-\infty, E)}(H)$, is exactly the number of zeros of $u(x, E)$ in $(0, \infty)$.

There is another distinct approach, essentially Sturm's approach in [22, to Sturm theory on the real line that we should mention. Consider 
zeros of $u(x, E)$, that is, solutions of

$$
u(x(E), E)=0
$$

$u$ is a $C^{1}$ function of $x$ and $E$, and if $u\left(x_{0}, E\right)=0$, then $u^{\prime}\left(x_{0}, E_{0}\right) \neq 0$ (since $u$ obeys a second-order ODE). Thus, by the implicit function theorem, for $E$ near $E_{0}$, there is a unique solution, $x(E)$, of (3.4) near $x_{0}$, and it obeys

$$
\left.\frac{d x}{d E}\right|_{E_{0}}=-\left.\frac{\partial u / \partial E}{\partial u / \partial x}\right|_{x=x_{0}, E=E_{0}}
$$

Now, $v \equiv \partial u / \partial E$ obeys the equation

$$
-v^{\prime \prime}+V v=E v+u
$$

by taking the derivative of $-u^{\prime \prime}+V u=E u$. Multiply (3.7) by $u$ and integrate by parts from 0 to $x_{0}$. Since $v(0)=0$, there is no boundary term at 0 , but there is at $x_{0}$, and we find

$$
v\left(x_{0}\right) u^{\prime}\left(x_{0}\right)=\int_{0}^{x_{0}}|u(x)|^{2} d x
$$

Thus (3.6) becomes

$$
\frac{d x_{0}}{d E}=-\left|u^{\prime}\left(x_{0}, E\right)\right|^{-2} \int_{0}^{x_{0}}|u(x, E)|^{2} d x<0
$$

Thus, as $E$ increases, zeros of $u$ move towards zero. This immediately implies the comparison theorem. Moreover, starting with $u_{n}$, the $(n+1)$-st eigenfunction at energy $E_{n}$, if it has $m$ zeros in $(0, a)$ as $E$ decreases from $E_{n}$ to a value, $E^{\prime}$ below $-\|V\|_{\infty}$ (where $u\left(x, E^{\prime}\right)>0$ has no zeros in $(0, \infty))$, the $m$ zeros move out continuously, and so $u(a, E)=0$ exactly $m$ times, that is, $m=n$. This proves the oscillation theorem.

\section{Rotation Numbers and Oscillations}

Take the solution $u(x, E)$ of the last section and look at the point

$$
\pi(x, E)=\left(\begin{array}{l}
u^{\prime}(x, E) \\
u(x, E)
\end{array}\right)
$$

in $\mathbb{R}^{2} . \pi$ is never zero since $u$ and $u^{\prime}$ have no common zeros. At most points in $\mathbb{R}^{2}$, the argument of $\pi$, that is, the angle $\pi$ makes with $\left(\begin{array}{l}1 \\ 0\end{array}\right)$, can increase or decrease. $u$ can wander around and around. But not at points where $u=0$. If $u^{\prime}>0$ at such a point, $\pi$ moves from the lower right quadrant to the upper right, and similarly, if $u^{\prime}<0$, it moves

from the upper left to lower left. Thus, since $\pi$ starts at $\left(\begin{array}{l}1 \\ 0\end{array}\right)$, we see 
Theorem 4.1. If $u(x, E)$ has $m$ zeros in $(0, a)$, then $\operatorname{Arg} \pi(a, E)$ (defined by continuity and $\operatorname{Arg} \pi(0, E)=0)$ lies in $\left(m \frac{\pi}{2},(m+1) \frac{\pi}{2}\right]$.

If $u$ and $v$ are two solutions of $-u^{\prime \prime}+V u=E u$ with $u(0)=0$, $v(0) \neq 0$, we can look at

$$
\tilde{\pi}(x, E)=\left(\begin{array}{l}
u \\
v
\end{array}\right)
$$

$\tilde{\pi}$ is never zero since $u$ and $v$ are linear independent. $W(x)=u^{\prime} v-v^{\prime} u$ is a constant, say $c . c \neq 0$ since $u$ and $v$ are linear independent. Suppose $c>0$. Then if $u\left(x_{0}\right)=0, u^{\prime}\left(x_{0}\right)=c / v\left(x_{0}\right)$ has the same sign as $v\left(x_{0}\right)$. So the above argument applies (if $c<0$, there is winding in the $(u, v)$ plane in the opposite direction). Rather than look at $\tilde{\pi}$, we can look at $\varphi=u+i v$. Then $u^{\prime} v-v u^{\prime}=\operatorname{Im}\left(\bar{\varphi} \varphi^{\prime}\right)$. Thus we have

Theorem 4.2. Let $\varphi(x, E)$ obey $-\varphi^{\prime \prime}+V \varphi=E \varphi$ and be complexvalued with

$$
\operatorname{Im}\left(\bar{\varphi}(0) \varphi^{\prime}(0)\right)>0
$$

Suppose $\operatorname{Re} \varphi(0)=0$. Then, if $\operatorname{Re} \varphi$ has $m$ zeros in $(0, a)$, then $\operatorname{Arg}(\varphi(a))$ is in $\left(m \frac{\pi}{2},(m+1) \frac{\pi}{2}\right]$.

The ideas of this section are the basis of the relation of rotation numbers and density of states used by Johnson-Moser 12 (see also [1]). We will use them as the starting point of the next section.

\section{Renormalized Oscillation Theory}

Consider $H=-\frac{d^{2}}{d x^{2}}+V$ on $[0, \infty)$ with $u(0)=0$ boundary conditions where, as usual, for simplicity, we suppose that $V$ is bounded. By Theorem [3.5 $\operatorname{dim} P_{(-\infty, E)}(H)$ is the number of zeros of $u(x, E)$ in $(0, \infty)$. If we want to know $\operatorname{dim} P_{\left[E_{1}, E_{2}\right)}(H)$, we can just subtract the number of zeros of $u\left(x, E_{1}\right)$ on $(0, \infty)$ from those of $u\left(x, E_{2}\right)$. At least, if $\operatorname{dim} P_{\left(-\infty, E_{2}\right)}(H)$ is finite, one can count just by subtracting. But if $\operatorname{dim} P_{\left(-\infty, E_{1}\right)}(H)=\infty$ while $\operatorname{dim} P_{\left[E_{1}, E_{2}\right)}$ is finite, both $u\left(x, E_{2}\right)$ and $u\left(x, E_{1}\right)$ have infinitely many zeros, and so subtraction requires regularization.

One might hope that

$$
\operatorname{dim} P_{\left[E_{1}, E_{2}\right)}(H)=\lim _{a \rightarrow \infty}\left(N\left(E_{2}, a\right)-N\left(E_{1}, a\right)\right)
$$

where $N(E, a)$ is the number of zeros of $u(x, E)$ in $(0, a)$. This is an approach of Hartmann 9]. (5.1) cannot literally be true since $N\left(E_{2}, a\right)-N\left(E_{1}, a\right)$ is an integer which clearly keeps changing when one passes through a zero of $u\left(x, E_{2}\right)$ that is not also a zero of $u\left(x, E_{1}\right)$. 
One can show that for a large, the absolute value of difference of the two sides of (5.1) is at most one, but it is not obvious when one has reached the asymptotic region.

Instead, we will describe an approach of Gesztesy, Simon, and Teschl [8] see Schmidt [19] for further discussion. Here it is for the half-line (the theorem is true in much greater generality than $V$ bounded and there are whole-line results).

Theorem 5.1. Let $V$ be bounded and let $H=-\frac{d^{2}}{d x^{2}}+V(x)$ on $[0, \infty)$ with $u(0)=0$ boundary conditions. Fix $E_{1}<E_{2}$. Let

$$
W(x)=u\left(x, E_{1}\right) u^{\prime}\left(x, E_{2}\right)-u^{\prime}\left(x, E_{1}\right) u\left(x, E_{2}\right)
$$

and let $N$ be the number of zeros of $W$ in $(0, \infty)$. Then

$$
\operatorname{dim} P_{\left(E_{1}, E_{2}\right)}(H)=N
$$

The rest of this section will sketch the proof of this theorem under the assumption that $\operatorname{dim} P_{\left(-\infty, E_{2}\right)}(H)=\infty$. This will allow a simplification of the argument and covers cases of greatest interest. Following [8], we will prove this in three steps:

(1) Prove the result in a finite interval $[0, a]$ in case $u\left(a, E_{2}\right)=0$.

(2) Prove $\operatorname{dim} P_{\left(E_{1}, E_{2}\right)}(H) \leq N$ by limits from (1) when $\operatorname{dim} P_{\left(-\infty, E_{2}\right)}(H)=\infty$.

(3) Prove $\operatorname{dim} P_{\left(E_{1}, E_{2}\right)}(H) \geq N$ by a variational argument.

Step 1. We use the rotation number picture of the last section. Define the Prüfer angle $\theta(x, E)$ by

$$
\tan (\theta(x, E))=\frac{u(x, E)}{u^{\prime}(x, E)}
$$

with $\theta(0, E)=0$ and $\theta$ continuous at points, $x_{0}$, where $u^{\prime}\left(x_{0}, E\right)=0$. Using $\frac{d}{d y} \tan y=1+\tan ^{2} y$, we get

$$
\frac{d \theta}{d x}=\frac{\left(u^{\prime}\right)^{2}-u u^{\prime \prime}}{u^{2}+\left(u^{\prime}\right)^{2}}
$$

Let $\theta_{1}, \theta_{2}$ be the Prüfer angles for $u_{1}(x) \equiv u\left(x, E_{1}\right)$ and $u_{2}(x) \equiv$ $u\left(x, E_{2}\right)$. Suppose $W\left(x_{0}\right)=0$. This happens if and only if $u\left(x_{0}, E\right) / u^{\prime}\left(x_{0}, E_{1}\right)=u\left(x_{0}, E_{2}\right) / u^{\prime}\left(x_{0}, E_{2}\right)$, that is, $\theta_{2}=\theta_{1}+k \pi$ with $k \in \mathbb{Z}$. If it happens, we can multiply $u_{2}$ by a constant so $u_{1}\left(x_{0}\right)=u_{2}\left(x_{0}\right), u_{1}^{\prime}\left(x_{0}\right)=u_{2}^{\prime}\left(x_{0}\right)$. Once we do that, (5.5) says

$$
\frac{d}{d x}\left(\theta_{2}-\theta_{1}\right)=\frac{\left(E_{2}-E_{1}\right) u_{1}^{2}\left(x_{0}\right)}{u_{1}^{\prime}\left(x_{0}\right)^{2}+u_{1}^{2}\left(x_{0}\right)}>0
$$

Thus

$$
\theta_{1}=\theta_{2} \quad \bmod \pi \Rightarrow \theta_{2}^{\prime}>\theta_{1}^{\prime}
$$


Think of $\theta_{2}$ as a hare and $\theta_{1}$ as a tortoise running around a track of length $\pi$. There are two rules in their race. They can each run in either direction, except they can only pass the starting gate going forward (i.e., $\theta_{j}=0 \bmod \pi \Rightarrow \theta_{j}^{\prime}>0$ ), and the hare can pass the tortoise, not vice-versa (i.e., (5.6) holds).

Suppose $H_{a}$, the operator on $(0, a)$ with $u(0)=u(a)=0$ boundary condition, has $m$ eigenvalues below $E_{2}$ and $n$ below $E_{1}$. Since $u\left(a, E_{2}\right)=0, \theta_{2}(a)=(m+1) \pi$, that is, at $x=a$, the hare makes exactly $m+1$ loops of the track. At $x=a$, the tortoise has made $n$ loops plus part, perhaps all, of an additional one. Since $\theta_{2}^{\prime}-\theta_{1}^{\prime}>0$ at $x=0$, the hare starts out ahead. Thus, the hare must overtake the tortoise exactly $m-n$ times between 0 and $a\left(\right.$ if $\theta_{1}(a)=(n+1) \pi$, since then $\theta_{2}^{\prime}-\theta_{1}^{\prime}>0$ at $x=0, \theta_{2}-(m+1) \pi<\theta_{1}-(n-1) \pi$, and $x=a$; so it is still true that there are exactly $m-n$ crossings). Thus

$$
P_{\left(E_{1}, E_{2}\right)}\left(H_{a}\right)=\#\left\{x_{0} \in(0, a) \mid W\left(x_{0}\right)=0\right\}
$$

Step 2. Since $\operatorname{dim} P_{\left(-\infty, E_{2}\right)}(H)=\infty$, there is, by Theorem [3.5] an infinite sequence $a_{1}<a_{2}<\cdots \rightarrow \infty$ so that $u\left(a_{j}, E_{2}\right)=0 . H_{a_{j}} \rightarrow H$ in strong resolvent sense, so by a simple argument,

$$
\begin{aligned}
\operatorname{dim} P_{\left(E_{1}, E_{2}\right)}(H) & \leq \lim \inf \operatorname{dim} P_{\left(E_{1}, E_{2}\right)}\left(H_{a}\right) \\
& =N
\end{aligned}
$$

with $N$ the number of zeros of $W$ in $(0, \infty)$. (5.8) comes from (5.7).

Step 3. Suppose $N<\infty$. Let $0<x_{1}<\cdots<x_{N}$ be the zeros of $W$. Define

$$
\begin{aligned}
& \eta_{j}(x)= \begin{cases}u_{1}(x)-\gamma_{j} u_{2}(x) & 0<x \leq x_{j} \\
0 & x \geq x_{j}\end{cases} \\
& \tilde{\eta}_{j}(x)= \begin{cases}u_{1}(x)+\gamma_{j} u_{2}(x) & 0<x<x_{j} \\
0 & x>x_{j}\end{cases}
\end{aligned}
$$

where $u_{j}(x)=u\left(x, E_{j}\right)$ and $\gamma_{j}$ is chosen by

$$
\gamma_{j}= \begin{cases}u_{1}\left(x_{j}\right) / u_{2}\left(x_{j}\right) & \text { if } u\left(x_{j}\right) \neq 0 \\ u_{1}^{\prime}\left(x_{j}\right) / u_{2}^{\prime}\left(x_{j}\right) & \text { if } u\left(x_{j}\right)=0\end{cases}
$$

Since $W\left(x_{j}\right)=0, \eta_{j}$ is a $C^{1}$ function of compact support and piecewise $C^{2}$, and so in $D(H)$. But $\tilde{\eta}$ is discontinuous. 
We claim that if $\eta$ is in the span of $\left\{\eta_{j}\right\}_{j=1}^{N}$, then

$$
\left\|\left(H-\frac{E_{2}+E_{1}}{2}\right) \eta\right\|=\frac{\left|E_{2}-E_{1}\right|}{2}\|\eta\|
$$

Moreover, such $\eta$ 's are never a finite linear combination of eigenfunctions of $H$. Accepting these two facts, we note that since the $\eta_{j}$ are obviously linear independent, (5.12) implies $\operatorname{dim} P_{\left(E_{1}, E_{2}\right)}(H) \geq N$. This, together with (5.8), proves the result.

To prove (5.12), we note that

$$
\left(H-\frac{E_{2}+E_{1}}{2}\right) \eta_{j}=-\frac{\left|E_{2}-E_{1}\right|}{2} \tilde{\eta}_{j}
$$

Since $\tilde{\eta}_{j}$ is not $C^{1}$ at $x_{j}$, no $\tilde{\eta}$ is in $D(H)$, hence no $\eta$ can be in $D\left(H^{2}\right)$ (so we get control of $\operatorname{dim} P_{\left(E_{1}, E_{2}\right)}(H)$, not just $\operatorname{dim} P_{\left[E_{1}, E_{2}\right]}(H)$ ).

Next, note that since $W^{\prime}(x)=\left(E_{2}-E_{1}\right) u_{2} u_{1}$, we have if $W\left(x_{i}\right)=$ $W\left(x_{i+1}\right)=0$ that

$$
\int_{x_{i}}^{x_{i+1}} u_{1}(x) u_{2}(x) d x=0
$$

for $i=0,1,2, \ldots, N$ where $x_{0}=0$. Thus

$$
\left\langle\eta_{i}, \eta_{j}\right\rangle=\left\langle\tilde{\eta}_{i}, \tilde{\eta}_{j}\right\rangle
$$

since if $i<j$, the difference of the two sides is $2\left(\gamma_{i}+\right.$ $\left.\gamma_{j}\right) \int_{x_{i}}^{x_{j}} u_{1}(x) u_{2}(x)=0$. (5.14) and (5.13) implies (5.12). That completes the proof if $N<\infty$.

If $N$ is infinite, pick $0<x_{1}<\cdots<x_{L}$ successive zeros and deduce $\operatorname{dim} P_{\left(E_{1}, E_{2}\right)}(H) \geq L$ for all $L$.

\section{Some Applications}

We will consider three typical applications in this section: one classical (i.e., fifty years old!), one recent to difference equations, and one of Theorem 5.1 .

Application 1: Bargmann's Bound. Let $u$ obey $-u^{\prime \prime}+V u=0$ with $u(0)=0$ so, if $V$ is bounded, $u(x) / x$ has a finite limit as $x \downarrow 0$. Also suppose $V \leq 0$.

Define $\tilde{m}=-u^{\prime} / u$ so

$$
\tilde{m}^{\prime}=|V|+\tilde{m}^{2}
$$

since $-V=|V|$. Thus $\tilde{m}$ is monotone increasing. It has a pole at each zero, $x_{0}=0, x_{1}, x_{2}, \ldots, x_{\ell}, \ldots$ of $u$. Define

$$
b(x)=-\frac{x u^{\prime}}{u}=x \tilde{m}(x)
$$


Then $b(x)$ has limit -1 as $x \downarrow 0$ and

$$
b^{\prime}(x)=x|V|+\frac{\left(b+b^{2}\right)}{x}
$$

In particular,

$$
-1 \leq b \leq 0 \Rightarrow b^{\prime}(x) \leq x|V|
$$

By the monotonicity of $\tilde{m}$, there are unique points $0<z_{1}<x_{1}<$ $\cdots<x_{\ell-1}<z_{\ell}<x_{\ell}$ where $b_{\ell}=0$, and since $b \rightarrow-\infty$ as $x \downarrow x_{j}$, there are last points $y_{j} \subset\left[x_{j-1}, z_{j}\right]$ where $b(y)=-1$ for $j=2,3, \ldots, \ell$ and at $y_{1}=0, b(0)=-1$. Integrating $b^{\prime}$ from $y_{j}$ to $z_{j}$, using (6.4), we find

$$
\int_{y_{j}}^{z_{j}} x|V(x)| d x \geq 1
$$

SO

$$
\int_{0}^{x_{\ell}} x|V(x)| d x \geq \ell
$$

By the oscillation theorem, if $N(V)=\operatorname{dim} P_{(-\infty, 0)}(H)$, then

$$
N(V) \leq \int_{0}^{\infty} x|V(x)| d x
$$

This is Bargmann's bound [1]. For further discussion, see Schmidt [20].

Application 2: Denisov-Rakhmanov Theorem. Rakhmanov 14, 15] (see also [13]) proved a deep theorem about orthogonal polynomials on the unit circle that translates to

Rakhmanov's Theorem. If $J$ is an infinite Jacobi matrix , $d \mu=$ $f d x+d \mu_{\mathrm{s}}$ and $f(x)>0$ and $x \in[-2,2]$ and $\operatorname{supp}\left(d \mu_{\mathrm{s}}\right) \subset[-2,2]$ (i.e., $\operatorname{spec}(J) \subset[-2,2])$, then $a_{n} \rightarrow 1, b_{n} \rightarrow 0$.

From the 1990's, there was some interest in extending this to the more general result, where $\operatorname{spec}(J) \subset[-2,2]$ is replaced by ess $\operatorname{spec}(J) \subset[-2,2]$. By using the ideas of the proof of Rakhmanov's theorem, one can prove:

Extended Rakhmanov Theorem. There exist $C(\varepsilon) \rightarrow 0$ as $\varepsilon \downarrow 0$ so that if $d \mu=f d x+d u$ and $f(x)>0$ a.e. $x$ in $[-2,2]$ and $\operatorname{spec}(J) \subset$ $[-2-\varepsilon, 2+C]$, then

$$
\lim \sup \left(\left|a_{n}-1\right|+\left|b_{n}\right|\right) \leq C(\varepsilon)
$$

Here is how Denisov [6] used this to prove

Denisov-Rakhmanov Theorem. If $d \mu=f(x) d x+d \mu_{0}, f(x)>0$ a.e. $x \in[-2,2]$ and $\sigma_{\mathrm{ess}}(J) \subset[-2,2]$, then $a_{n} \rightarrow 1$ and $b_{n} \rightarrow 0$. 
His proof goes as follows. Fix $\varepsilon$. Since $J$ has only finitely many eigenvalues in $[2+\varepsilon, \infty), P_{n}(2+\varepsilon)$ has only finitely many sign changes. Similarly, $(-1)^{n} P_{n}(-2-\varepsilon)$ has only finitely many sign changes. Thus, we can find $N_{0}$ so $P_{n}(2+\varepsilon)$ and $(-1)^{n} P_{n}(-2-\varepsilon)$ both have fixed signs if $n>N_{0}$. Let $\tilde{a}, \tilde{b}$ be given by

$$
\tilde{a}_{n}=a_{N_{0}+n} \quad \tilde{b}_{n}=b_{N_{0}+n}
$$

By a use of the comparison and oscillation theorems, $\tilde{J}$ has no eigenvalues in $(-\infty,-2-\varepsilon) \cup(2+\varepsilon, \infty)$. Thus, by the Extended Rakhmanov Theorem,

$$
\limsup \left(\left|a_{n}-1\right|+\left|b_{n}\right|\right)=\lim \sup \left(\left|\tilde{a}_{n}-1\right|+\left|\tilde{b}_{n}\right|\right) \leq C(\varepsilon)
$$

Since $\varepsilon$ is arbitrary, the theorem is proven.

Application 3: Teschl's Proof of the Rofe-Beketov Theorem. Let $V_{0}(x)$ be periodic and continuous. Let $H_{0}=-\frac{d^{2}}{d x^{2}}+V_{0}$ on $L^{2}(0, \infty)$ with $u(0)=0$ boundary condition. Then

$$
\sigma_{\mathrm{ess}}\left(H_{0}\right)=\bigcup_{j=1}^{\infty}\left[a_{j}, b_{j}\right]
$$

with $b_{j}<a_{j+1}$. (In some special cases, there is only a finite union with one infinite interval.) $\left(b_{j}, a_{j+1}\right)$ are called the gaps. In each gap, $H_{0}$ has either zero or one eigenvalue. Suppose $X(x) \rightarrow 0$ as $x \rightarrow \infty$, and let $H=H_{0}+X$. Since $\sigma_{\text {ess }}(H)=\sigma_{\text {ess }}\left(H_{0}\right), H$ also has gaps in its spectrum. When is it true that each gap has at most finitely many eigenvalues? Teschl [24, 25] has proven that if $\int_{0}^{\infty} x|X(x)| d x<\infty$, then for each $j$, the Wronskian, $w(x)$, of $u\left(x, b_{j}\right)$ and $u\left(x, a_{j+1}\right)$ has only finitely many zeros. He does this by showing for $H_{0}$ that $|X(x)| \rightarrow \infty$ as $x \rightarrow \infty$ and by an ODE perturbation argument, this implies $|w(x)| \rightarrow \infty$ for $H$. Thus, by the results of Section 5 , there are finitely many eigenvalues in each gap.

It is easy to go from half-line results to whole-line results, so Teschl proves if $\int|x||X(x)| d x<\infty$, each gap has only finitely many eigenvalues.

This result was first proven by Rofe-Beketov [18] with another simple proof in Gesztesy-Simon [7]; see that later paper for additional references. Teschl's results are stated for the discrete (Jacobi) case (and may be the first proof for the finite difference situation), but his argument translates to the one above for Schrödinger operators. 


\section{REFERENCES}

[1] V. Bargmann, On the number of bound states in a central field of force, Proc. Nat. Acad. Sci. U.S.A. 38 (1952), 961-966.

[2] M. Bôcher, The theorems of oscillation of Sturm and Klein, Bull. Amer. Math. Soc. 4 (1897-1898), 295-313, 365-376.

[3] E.A. Coddington and N. Levinson, Theory of Ordinary Differential Equations, McGraw-Hill, New York-Toronto-London, 1955.

[4] R. Courant and D. Hilbert, Methods of Mathematical Physics, Vol. I, Interscience Publishers, New York, N.Y., 1953.

[5] F. Delyon and B. Souillard, The rotation number for finite difference operators and its properties, Comm. Math. Phys. 89 (1983), 415-426.

[6] S.A. Denisov, On Rakhmanov's theorem for Jacobi matrices, to appear in Proc. Amer. Math. Soc.

[7] F. Gesztesy and B. Simon, A short proof of Zheludev's theorem, Trans. Amer. Math. Soc. 335 (1993), 329-340.

[8] F. Gesztesy, B. Simon, and G. Teschl, Zeros of the Wronskian and renormalized oscillation theory, Amer. J. Math. 118 (1996), 571-594.

[9] P. Hartman, Uniqueness of principal values, complete monotonicity of logarithmic derivatives of principal solutions, and oscillation theorems, Math. Ann. 241 (1979), 257-281.

[10] I. James, Remarkable Mathematicians. From Euler to von Neumann, Mathematical Association of America, Washington, DC; Cambridge University Press, Cambridge, 2002.

[11] R. Johnson, Oscillation theory and the density of states for the Schrödinger operator in odd dimension, J. Differential Equations 92 (1991), 145-162.

[12] R. Johnson and J. Moser, The rotation number for almost periodic potentials, Comm. Math. Phys. 84 (1982), 403-438.

[13] A. Máté, P. Nevai, and V. Totik, Asymptotics for the ratio of leading coefficients of orthonormal polynomials on the unit circle, Constr. Approx. 1 (1985), 63-69.

[14] E.A. Rakhmanov, On the asymptotics of the ratio of orthogonal polynomials, Math. USSR Sb. 32 (1977), 199-213.

[15] E.A. Rakhmanov, Asymptotic properties of polynomials orthogonal on the circle with weights not satisfying the Szegö condition, Math. USSR-Sb. $\mathbf{5 8}$ (1987), 149-167; Russian original in Mat. Sb. (N.S.) 130(172) (1986), 151$169,284$.

[16] M. Reed and B. Simon, Methods of Modern Mathematical Physics, Vol. 1: Functional Analysis, Academic Press, New York, 1972.

[17] M. Reed and B. Simon, Methods of Modern Mathematical Physics, II. Fourier Analysis, Self-Adjointness, Academic Press, New York, 1975.

[18] F.S. Rofe-Beketov, Perturbation of a Hill operator having a first moment and nonzero integral creates one discrete level in distant spectral gaps, Mat. Fizika i Funkts. Analiz. (Kharkov) 19 (1973), 158-159. [Russian]

[19] K.M. Schmidt, An application of the Gesztesy-Simon-Teschl oscillation theory to a problem in differential geometry, J. Math. Anal. Appl. 261 (2001), 61-71.

[20] K.M. Schmidt, A short proof for Bargmann-type inequalities, Proc. Roy. Soc. London. Ser. A 458 (2002), 2829-2832. 
[21] B. Simon, The classical moment problem as a self-adjoint finite difference operator, Adv. Math. 137 (1998), 82-203.

[22] C. Sturm, Sur les équations différentielles linéaires du second ordre, J. Math. Pures et Appl. de Liouville 1 (1836), 106-186.

[23] C. Sturm, Sur une classe d'équations différentielles partielles, J. Math. Pures et Appl. de Liouville 1 (1836), 375-444.

[24] G. Teschl, Oscillation theory and renormalized oscillation theory for Jacobi operators, J. Differential Equations 129 (1996), 532-558.

[25] G. Teschl, Jacobi Operators and Completely Integrable Nonlinear Lattices, Mathematical Surveys and Monographs, 72, American Mathematical Society, Providence, R.I., 2000. 